\title{
Performance of Dual-Hop Multi-Antenna Systems with Fixed Gain Amplify-and-Forward Relay Selection
}

\author{
Shiva Prakash, Member, IEEE, Ian McLoughlin, Senior Member, IEEE
}

\begin{abstract}
The performance of amplify-and-forward relaying in Rayleigh channels is explored for a dual-hop transmission system in which a transmission source selects one of several relays based upon instantaneous SNR (signal to noise ratio) to transmit a data packet to a destination receiver. Both source and receiver devices have multiple antennas, employing beamforming for transmission and MRC (maximal ratio combining) for reception. The chosen relay performs fixed gain forwarding. Closed form solutions for several performance measures are derived for this practical system, and the system is studied in terms of outage probability and symbol error rate which are verified through simulation. Several implementation alternatives are explored to note performance trade-offs, particularly between number of antennas and number of relays.
\end{abstract}

Index Terms-Cooperative diversity, relay selection, amplifyand -forward, beamforming, symbol error rate, outage probability.

\section{INTRODUCTION}

$\mathbf{R}$ ELAYING may be useful in future wireless systems such as $4 \mathrm{G}$ and LTE (long term evolution) that demand high data rates and spectral efficiencies[1]. Co-operative relaying is known to be capable of improving throughput and coverage, plus better utilise resources such as bandwidth and power[1], [2]. This letter explores a system in which a multi antenna source and destination communicate with the aid of an intermediate, single antenna, relay which is selected from several candidates by the transmitter. We use the less complex non-regenerative amplify-and-forward (AF) relay [3] rather than a regenerative system which would fully decode the data packet at the selected relay prior to transmission over the second hop. Transmit-directed relay selection, based on instantaneous channel measurement, is employed- a low complexity and efficient method of harnessing distributed spatial diversity across a co-operative virtual antenna array[4]. Previous systems (including Zhao[5], who studied the performance benefits of best-relay offering maximum SNR gain across both hops), are limited to single antenna nodes. Partial relay selection, as proposed by Krikidis et al. [6], alleviates the overhead of complete CSI (channel state information) being transmitted across both hops (this assumed variable relay gain). This has advantages in prolonging network lifetime. Recently, Suraweera et al.[7], and DaCosta and Aissa [8], [9]

Manuscript received August 7, 2010; revised November 21, 2010; accepted January 30, 2011. The associate editor coordinating the review of this letter and approving it for publication was Dr. Aydin.

The authors are with the School of Computer Engineering, Nanyang Technological University, 50 Nanyang Avenue, Singapore (e-mail: shiv0008@e.ntu.edu.sg, mcloughlin@ntu.edu.sg).

Digital Object Identifier 10.1109/TWC.2011.101415.

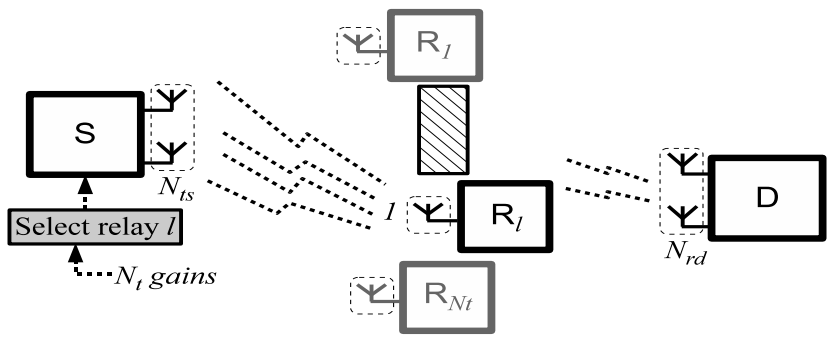

Fig. 1: Dual-hop relay selection system equipped with multiple antennas at source and destination.

studied fixed gain AF with first hop relay selection. However, both systems where restricted to single antenna terminals at the relay nodes and destination. Interestingly, performance was not found to increase appreciably when the number of relay terminals increases. Instead, gains were limited by the quality of the non-selective second hop from relay to destination. Very little work has been published concerning fixed-gain relaying in multi-antenna systems, however source beamforming has been studied[10] for single antenna relay (with variable gain) and single antenna destination, in which it was observed that at high SNR employing more than two transmit antennas did not sufficiently improve outage probability performance. In [11], multiple destination receive antennas in conjunction with source beamforming was analysed, using the moment generating function approach to analyse BER (bit error rate) performance. However, increasing the transmit or receive antennas at either source or destination end to boost performance may not be feasible in many situations; and in some cases, use of additional antennas might prove detrimental to overall improvement. Keeping in mind the drawbacks of the previous schemes and the attractive simplicity of selection, this paper considers a beamforming system for Rayleigh fading channels with multiple relay nodes, each equipped with a single antenna, as in Fig. 1. Best relay selection, based on instantaneous SNRs, is made at the transmitter and, to prevent performance saturation over the first hop, the second hop link is strengthened via multiple receive antennas at the destination. We will show that when the number of transmit (at source) or receive (at destination) antennas is to be kept small, use of multiple intermediate relays to improve performance is a potential solution. Likewise, when the number of relays are limited, deploying multiple antennas at source or destination can improve performance. Importantly, it will be shown that a wise selection of network parameters (through number of transmit, receive antennas or number of relays) is important to realize large gains in such a system. We study this system in terms of outage probability $\left(P_{\text {out }}\right)$ and symbol error rate 
(SER), and derive the moment generating function (MGF), statistical moments of the end-to-end SNR. Since the relays are assumed to be blind or semi-blind to CSI, they offer only fixed amplification to the incoming signal- easier to implement than a variable gain AF system, at the expense of some small performance loss. We also derive the amplification gain for the semi-blind scenario.

\section{SYSTEM MODEL}

We consider a relay network, as in Fig. 1, where source $S$ equipped with $N_{t s}$ transmit antennas, communicates with relatively closely spaced $N_{t}$ single antenna relay nodes $R_{1}, R_{2}, \ldots, R_{N_{t}}$. In the absence of a direct path, a single relay selected by the transmitter, relays data to destination $D$ which is equipped with $N_{r d}$ receive antennas. Based on pilot data broadcast by the relays, instantaneous CSI is measured at the source itself, thus alleviating the need for a feedback channel. The channel matrix at $S$ during initial channel estimation is denoted as $\mathbf{H}$, of dimension $N_{t s} \times N_{t}$ with complex fading coefficients $h_{i j}, 1 \leq i \leq N_{t s}, 1 \leq j \leq N_{t}$ as its entries. The channels $h_{i j}$ are characterised as spatially independent and identically distributed (i.i.d) complex Gaussian random variables with distribution $\mathcal{C N}(0,1)$. Also, they change slowly enough that multiple training signals can be transmitted without significant frame overhead. The source computes the instantaneous channel power for each relay as $p_{j}=\sum_{i=0}^{N_{t s}}\left|h_{i j}\right|^{2}$ and picks a single best relay $1 \leq l \leq N_{t}$, corresponding to the maximum power: $p_{\max }=\max _{1 \leq j \leq N_{t}} p_{j}$. The first transmission hop to this relay, denoted as $S \rightarrow R_{l}$, has channel $\mathbf{h}_{S R_{l}}$. This is the $\left(N_{t s} \times 1\right)$ channel vector being the $l^{\text {th }}$ column of channel matrix $\mathbf{H}$, assumed to be correctly estimated at $S$. To perform transmit diversity beamforming, $S$ computes an $\left(1 \times N_{t s}\right)$ optimal beamforming vector [12] $\mathbf{w}_{S R_{l}}=\frac{\mathbf{h}_{S R_{l}}^{H}}{\sqrt{\mathbf{h}_{S R_{l}}^{H} \mathbf{h}_{S R_{l}}}}$ after which data is transmitted on the $S \rightarrow R_{l}$ channel. (. $)^{H}$ is the Hermitian transpose. The signal received at the relay is amplified by fixed gain $G$, and transmitted to $D$ via the $R_{l} \rightarrow D$ channel $\mathbf{h}_{R_{l} D}$. This is a $\left(N_{r d} \times 1\right)$ channel vector which is $\mathcal{C N}\left(0, \mathbf{I}_{N_{r d}}\right)$ distributed, also assumed to be estimated correctly at $D$ (I is an identity matrix). Here, the signal is finally demodulated, employing MRC [13] at the receiver with $\mathbf{w}_{R_{l} D}=\frac{\mathbf{h}_{R_{l} D}^{H}}{\sqrt{\mathbf{h}_{R_{l} D}^{H} \mathbf{h}_{R_{l} D}}}$ as the $\left(1 \times N_{r d}\right)$ optimal MRC weight vector at the destination. Then the final combined output at the destination is:

$$
y_{R_{l} D}=G \mathbf{w}_{R_{l} D} \mathbf{h}_{R_{l} D}\left(s \mathbf{w}_{S R_{l}} \mathbf{h}_{S R_{l}}+n_{R_{l}}\right)+\mathbf{w}_{R_{l} D} \mathbf{n}_{D}
$$

where $s$ is the transmitted symbol, $n_{R_{l}}$ is the AWGN noise sample at the relay characterised as $\mathcal{C N}\left(0, N_{0}\right)$, while $\mathbf{n}_{D}$ is the noise vector modelled as $\mathcal{C N}\left(0, N_{0} \mathbf{I}_{N_{r d}}\right)$ where $N_{0}$ is the variance of the AWGN (additive white Gaussian noise). The equivalent SNR at the destination is then calculated as:

$$
\gamma_{e q}=\frac{\frac{\mathcal{E}_{1} \mathbf{h}_{S R_{l}}^{H} \mathbf{h}_{S R_{l}}}{N_{0}} \frac{\mathcal{E}_{2} \mathbf{h}_{R_{l} D}^{H} \mathbf{h}_{R_{l} D}}{N_{0}}}{\frac{\mathcal{E}_{2}}{N_{0} G^{2}}+\frac{\mathcal{E}_{2} \mathbf{h}_{R_{l} D}^{H} \mathbf{h}_{R_{l} D}}{N_{0}}}
$$

$\mathcal{E}_{1}$ and $\mathcal{E}_{2}$ are the powers of the transmitted signal at the source and relay. If we keep $C=\frac{\mathcal{E}_{2}}{N_{0} G^{2}}$ constant for fixed gain $G$, then (2) can be simplified as:

$$
\gamma_{e q}=\gamma_{1} \gamma_{2} /\left(\gamma_{2}+C\right)
$$

where $\gamma_{1}=\frac{\mathcal{E}_{1} \mathbf{h}_{S R_{l}}^{H} \mathbf{h}_{S R_{l}}}{N_{0}}$ and $\gamma_{2}=\frac{\mathcal{E}_{2} \mathbf{h}_{R_{l} D}^{H} \mathbf{h}_{R_{l} D}}{N_{0}}$ represent the instantaneous SNRs of the first and second hops respectively. The average receive SNR for each diversity path in the first and second hop are denoted as $\bar{\gamma}_{1}=\frac{\mathcal{E}_{1}}{N_{0}}$ and $\bar{\gamma}_{2}=\frac{\mathcal{E}_{2}}{N_{0}}$ respectively.

\section{Performance Metrics}

In the section we will first formulate the statistics of each hop and use these to derive precise expressions for important performance measures such as $P_{\text {out }}$, SER, MGF and moments of end-to-end SNR $\gamma_{e q}$. Additionally, we also derive the gain $G$ in a semi-blind relay scenario, where the relays have partial or statistical information about the first hop channel.

\section{A. Outage Probability}

The outage probability $P_{\text {out }}$ at any given average received SNR is defined as the probability that the instantaneous SNR is less than a threshold, $\gamma_{T}$. In the relay system under consideration this is expressed as [3]:

$$
\begin{aligned}
P_{\text {out }}\left(\gamma_{T}\right) & =P_{\text {out }}\left(\gamma_{\text {eq }}<\gamma_{T}\right) \\
& =\int_{0}^{\infty} \operatorname{Pr}\left[\frac{\gamma_{1} \gamma_{2}}{\gamma_{2}+C}<\gamma_{T} \mid \gamma_{2}\right] f_{\gamma_{2}}\left(\gamma_{2}\right) d \gamma_{2}
\end{aligned}
$$

where $f_{\gamma_{2}}\left(\gamma_{2}\right)$ is the PDF of the $R_{l} \rightarrow D$ channel. To solve (4) we first need $F_{\gamma_{1}}($.$) , the CDF of \gamma_{1}$, so that firstly, the inner probability term is solved. Making use of order statistics[14, p.246], the corresponding PDF $f_{\gamma_{1}}(\gamma)$ can be expressed as follows:

$$
f_{\gamma_{1}}(\gamma)=N_{t} f_{\gamma_{0}}(\gamma)\left[F_{\gamma_{0}}(\gamma)\right]^{N_{t}-1}
$$

where $f_{\gamma_{0}}(\gamma)$ is the PDF of the SNRs of the individual and independent $S \rightarrow R_{i=1 \ldots, N_{t}}$ links, characterised by a gamma PDF $\mathcal{G} \backsim\left(N_{t s}, \bar{\gamma}_{1}\right)$ given by

$$
f_{\gamma_{0}}(\gamma)=\frac{\gamma^{N_{t s}-1}}{{\overline{\gamma_{1}}}^{N_{t s}}\left(N_{t s}-1\right) !} \exp \left(-\frac{\gamma}{\overline{\gamma_{1}}}\right)
$$

with their $\mathrm{CDF}$ given as:

$$
F_{\gamma_{0}}(\gamma)=1-\exp \left(-\gamma / \overline{\gamma_{1}}\right) \sum_{j=0}^{N_{t_{s}}-1}(1 / j !)\left(\gamma / \overline{\gamma_{1}}\right)^{j}
$$

To obtain a series expansion of (5), $\left[F_{\gamma_{0}}(\gamma)\right]^{N_{t}-1}$ is expanded first via the binomial theorem followed by multinomial theorem, and coupled with (6). Then, grouping exponential terms and powers of $\gamma$, the definite integral of (5) is then solved to obtain $F_{\gamma_{1}}(\cdot)$ as follows:

$$
\begin{aligned}
& F_{\gamma_{1}}(z)=\int_{0}^{z} f_{\gamma_{1}}(\gamma) d \gamma \\
&=\frac{N_{t} !}{\bar{\gamma}_{1}^{N_{t s}}\left(N_{t s}-1\right) !} \sum_{i=0}^{N_{t}-1} \frac{(-1)^{i}}{i !\left(N_{t}-1-i\right) !} \\
& \sum_{j=0}^{i\left(N_{t s}-1\right)} \eta_{N_{t s}}(i, j)\left(1 / \overline{\gamma_{1}}\right)^{j} \int_{0}^{z} \gamma^{j+N_{t s}-1} \exp \left(-(i+1) \gamma / \bar{\gamma}_{1}\right) d \gamma
\end{aligned}
$$


where $z$ is a constant and $\eta_{N_{t s}}(i, j)[15,0.314]$ are the coefficients of $\varphi^{j}, j=0,1, \ldots i\left(N_{t s}-1\right)$ in the expansion of $\left(\sum_{j=0}^{N_{t s}-1} \varphi^{j} / j !\right)^{i}$, used in the expansion of $\left[F_{\gamma_{0}}(\gamma)\right]^{N_{t}-1}$. Finally, using [15, eqn.(3.381.1)], (8) is solved and $F_{\gamma_{1}}(z)$ is cast in a complimentary CDF form as follows:

$$
\begin{aligned}
& F_{\gamma_{1}}(z)=1-\frac{N_{t} !}{\left(N_{t s}-1\right) !} \sum_{i=0}^{N_{t}-1} \frac{(-1)^{i} \exp \left(-(i+1) z / \bar{\gamma}_{1}\right)}{i !\left(N_{t}-1-i\right) !} \\
& \sum_{j=0}^{i\left(N_{t s}-1\right)} \eta_{N_{t s}}(i, j)\left(N_{t s}+j-1\right) ! \sum_{l=0}^{j+N_{t s}-1} \frac{z^{l}}{\bar{\gamma}_{1}^{l} l !(i+1)^{j+N_{t s}-l}}
\end{aligned}
$$

Moving our focus back to solving (4), we first substitute $z=$ $\gamma_{T}\left(1+\frac{C}{\gamma_{2}}\right)$ in (9) to obtain $\operatorname{Pr}\left[\frac{\gamma_{1} \gamma_{2}}{\gamma_{2}+C}<\gamma_{T} \mid \gamma_{2}\right]$. Then a binomial expansion of $\left(1+C / \gamma_{2}\right)^{l}$ is done. Together, this is then coupled with $f_{\gamma_{2}}\left(\gamma_{2}\right)$, the PDF of the $R_{l} \rightarrow D$ which is $\mathcal{G} \backsim\left(N_{r d}, \bar{\gamma}_{2}\right)$ :

$$
f_{\gamma_{2}}\left(\gamma_{2}\right)=\frac{\gamma_{2}{ }^{N_{r d}-1}}{{\overline{\gamma_{2}}}^{N_{r d}}\left(N_{r d}-1\right) !} \exp \left(-\frac{\gamma_{2}}{\overline{\gamma_{2}}}\right)
$$

Collecting the power and exponential terms of $\gamma_{2}$, the integration of (4) is facilitated using [15, eqn.(3.471.9)]. Thus $P_{\text {out }}$ (or equivalently $F_{\gamma_{e q}}\left(\gamma_{T}\right)$ : the $\mathrm{CDF}$ of $\left.\gamma_{e q}\right)$ is obtained as:

$$
\begin{aligned}
& P_{\text {out }}\left(\overline{\gamma_{1}}, \overline{\gamma_{2}}, N_{t}, N_{t s}, N_{r d}, \gamma_{T}\right) \\
& =\int_{0}^{\infty} F_{\gamma_{1}}\left[\gamma_{T}\left(1+C / \gamma_{2}\right)\right] f_{\gamma_{2}}\left(\gamma_{2}\right) d \gamma_{2} \\
& =1-\frac{N_{t} !}{\left(N_{t s}-1\right) !\left(N_{r d}-1\right) ! \bar{\gamma}_{2}^{N_{r d}}} \sum_{i=0}^{N_{t}-1} \frac{(-1)^{i}}{i !\left(N_{t}-1-i\right) !} \\
& \cdot \sum_{j=0}^{i\left(N_{t s}-1\right)} \eta_{N_{t s}}(i, j)\left(N_{t s}+j-1\right) ! \sum_{l=0}^{j+N_{t s}-1} \frac{2}{(i+1)^{j+N_{t s}-l} l !} \\
& \cdot\left(\frac{\gamma_{T}}{\bar{\gamma}_{1}}\right)^{l} \exp \left(-\frac{\gamma_{T}(i+1)}{\bar{\gamma}_{1}}\right) \sum_{p=0}^{l}\left(\begin{array}{l}
l \\
p
\end{array}\right) C^{p} \\
& \cdot\left[\frac{C \gamma_{T}(i+1) \bar{\gamma}_{2}}{\bar{\gamma}_{1}}\right]^{\frac{\left(N_{r d}-p\right)}{2}} \mathbf{K}_{N_{r d}-p}\left(2 \sqrt{\frac{\gamma_{T} C(i+1)}{\bar{\gamma}_{1} \bar{\gamma}_{2}}}\right)
\end{aligned}
$$

where $\mathbf{K}_{v}(\cdot)$ is the modified Bessel function of the second kind of order $v$ [15]. As a check, for the simplified case of $N_{t s}=N_{r d}=N_{t}=1$, (11) reduces to [3, eqn.(9)].

\section{B. Error probability}

The mean symbol error rate (SER) at an average SNR is found by averaging the probability of symbol error in AWGN over the fading distribution $f_{\gamma_{e q}}(\gamma)$ of the relay scheme, in slow fading scenarios. SER for coherent demodulation can be found from:

$$
\bar{P}_{s}\left(\bar{\gamma}_{1}, \overline{\gamma_{2}}, N_{t}, N_{t s}, N_{r d}\right)=\int_{0}^{\infty} P_{s}(\gamma) f_{\gamma_{e q}}(\gamma) d \gamma
$$

where $P_{s}(\gamma)=\alpha Q(\sqrt{\beta \gamma}) . \alpha$ and $\beta$ are constellation specific constants and $Q(\cdot)$ is the $Q$-function [16]. With $\alpha=1, \beta=$ 2 , an exact BER solution is found for BPSK/ QPSK, while approximate (but accurate) SER for the spectrally efficient $M$ QAM (quadrature amplitude modulation) is obtained with $\alpha=$ $\frac{4(\sqrt{M}-1)}{\sqrt{M}}$ and $\beta=\frac{3}{(M-1)}$. Eqn. (12) can also be expressed using $F_{\gamma_{e q}}(\cdot)$ [5], so that $\bar{P}_{s}$ is derived without having to obtain the PDF $f_{\gamma_{e q}}(\cdot)$ :

$$
\begin{aligned}
& \bar{P}_{s}\left(\overline{\gamma_{1}}, \overline{\gamma_{2}}, N_{t s}, N_{t}, N_{r d}, \alpha, \beta\right)= \\
& \frac{\alpha}{\sqrt{(2 \pi)}} \int_{0}^{\infty} F_{\gamma_{e q}}\left(x^{2} / \beta\right) \exp \left(-x^{2} / 2\right) d x
\end{aligned}
$$

Using $\gamma_{T}=x^{2} / \beta$ in (11) and grouping powers of $x$, and the exponential term with $\exp \left(-x^{2} / 2\right)$ in (13), an integral containing a combination of power, exponential, and Bessel function is solved using [15, eqn.(6.631.3)]. Thus the SER of the desired modulation scheme under relaying can be evaluated as :

$$
\begin{aligned}
& \bar{P}_{s}\left(\overline{\gamma_{1}}, \overline{\gamma_{2}}, N_{t}, N_{t s}, N_{r d}, \alpha, \beta\right) \\
& =\frac{\alpha}{2}\left[1-\frac{N_{t} ! \bar{\gamma}_{2}^{-N_{r d}}}{\sqrt{2 \pi}\left(N_{t s}-1\right) !\left(N_{r d}-1\right) !} \sum_{i=0}^{N_{t}-1} \frac{(-1)^{i}}{i !\left(N_{t}-1-i\right) !}\right. \\
& \cdot \sum_{j=0}^{i\left(N_{t s}-1\right)} \eta_{N_{t s}}(i, j)\left(N_{t s}+j-1\right) ! \sum_{l=0}^{j+N_{t s}-1} \frac{1}{(i+1)^{j+N t s-l} l !} \\
& \cdot\left(\frac{1}{\beta \bar{\gamma}_{1}}\right)^{l} \sum_{p=0}^{l}\left(\begin{array}{l}
l \\
p
\end{array}\right) C^{p}\left(a_{i} C \bar{\gamma}_{2}\right)^{\left(N_{r d}-p\right) / 2} \\
& \cdot \boldsymbol{\Gamma}[l+1 / 2] \boldsymbol{\Gamma}\left[l+N_{r d}-p+1 / 2\right] \\
& \cdot \exp \left(\frac{b_{i}}{2 a_{i}+1}\right) \frac{\left(a_{i}+1 / 2\right)^{\left(p-2 l-N_{r d}\right) / 2}}{\sqrt{b_{i}}} \\
& \left.\cdot \mathbf{W}_{\left(p-2 l-N_{r d}\right) / 2,\left(N_{r d}-p\right) / 2}\left(\frac{b_{i}}{a_{i}+1 / 2}\right)\right]
\end{aligned}
$$

where $b_{i}=\frac{C(i+1)}{\bar{\gamma}_{1} \bar{\gamma}_{2} \beta}, a_{i}=\frac{(i+1)}{\bar{\gamma}_{1} \beta}, \Gamma[\cdot]$ is the gamma function. $\mathbf{W}_{k, \mu}(\cdot)$ is the Whittaker function [15] which can be evaluated in terms of the Bessel or hypergeometric ${ }_{2} \mathbf{F}_{0}(a, b ; z)$ function.

As a check, we set $N_{t s}=N_{r d}=1$; simplifying (14) and expanding $\mathbf{W}_{k, \mu}(\cdot)$, now as a series of Bessel $\mathbf{K}_{v}(\cdot)$ functions[17], where $k=-1 / 2$ and $\mu=1 / 2$ we can reduce (14) to [7, eqn.(12)]: the case for $N_{t}$ relay nodes but source and destinations with only single antennas.

Moment Generating Function: The MGF approach[18] is a powerful tool for evaluating the SER for a wide variety of $M$-ary modulations such as the $M$-ary phase shift keying ( $M$-PSK), $M$-ary differential phase shift keying ( $M$-DPSK) and $M$-QAM. The MGF of $\gamma_{e q}$ is given by $\mathcal{M}_{\gamma_{e q}}(s)=$ $\mathbb{E}\left[\exp \left(-s \gamma_{e q}\right)\right], \mathbb{E}[\cdot]$ is the expectation operator. For example, it is well known that the BER for binary DPSK is $\bar{P}_{s}=$ $0.5 \mathcal{M}_{\gamma_{e q}}(1)$. It is convenient to express $\mathcal{M}_{\gamma_{e q}}(s)$ as a integral containing the complimentary CDF, $1-F_{\gamma_{e q}}(\gamma)$, so that the tedious differentiation step to obtain the PDF $f_{\gamma_{e q}}(\gamma)$ from (11) can be skipped. Using integration by parts, it can be shown that:

$$
\mathcal{M}_{\gamma_{e q}}(s)=1-s \int_{0}^{\infty} \exp \left(-\gamma_{e q} s\right)\left(1-F_{\gamma_{e q}}(\gamma)\right) d \gamma
$$


Using $F_{\gamma_{e q}}(\cdot)$ from (11) in the above equation and [15, eqn.(6.643.3)], and after simplifications the closed form solution for the MGF can be written as:

$$
\begin{gathered}
\mathcal{M}_{\gamma_{e q}}(s)=1-\frac{s N_{t} ! \bar{\gamma}_{1}}{\left(N_{t s}-1\right) !\left(N_{r d}-1\right) !} \sum_{i=0}^{N_{t}-1} \frac{(-1)^{i}}{i !\left(N_{t}-1-i\right) !} \\
\cdot \exp \left(\frac{C(i+1)}{2 \bar{\gamma}_{2}\left(\bar{\gamma}_{1} s+i+1\right)}\right) \sum_{j=0}^{i\left(N_{t s}-1\right)} \eta_{N_{t s}}(i, j)\left(N_{t s}+j-1\right) ! \\
\cdot \sum_{l=0}^{j+N_{t s}-1} \frac{1}{l(i+1)^{j+N_{t s}-l}} \\
\cdot \sum_{p=0}^{l}\left(\begin{array}{l}
l \\
p
\end{array}\right) \frac{C^{\frac{N_{r d}+p-1}{2}}(i+1)^{\frac{N_{r d}-p-1}{2}}}{\left(\bar{\gamma}_{1} s+i+1\right)^{\frac{2 l+N_{r d}-p-1}{2}}} \frac{\Gamma\left[N_{r d}-p+l+1\right]}{\bar{\gamma}_{2}^{\left(N_{r d}+p-1\right) / 2}} \\
\cdot \mathbf{W}_{-\left(2 l+N_{r d}-p+1\right) / 2,\left(N_{r d}-p\right) / 2}\left(\frac{C(i+1)}{\bar{\gamma}_{2}\left(\bar{\gamma}_{1} s+i+1\right)}\right)
\end{gathered}
$$

As a check consider selection among $N_{t}$ single antenna terminals with $N_{t s}=N_{r d}=1$. Knowing that $W_{-\mu-1 / 2, \mu}(z)=$ $\exp (z / 2) z^{\frac{1}{2}-\mu} \boldsymbol{\Gamma}(-2 \mu, z)=\exp (z / 2) z^{\frac{1}{2}-\mu} E_{2 \mu+1}(z)$, where $E_{n}(z)$ is the exponential integral[15, p.xxxv], we can reduce the MGF to exactly [8, eqn.(22)]. While for $N_{t s}=N_{t}=$ $N_{r d}=1$, using the recurrence relation of $E_{n}(z)$, this simplifies to [3, eqn.(12)].

\section{Choice of gain parameter}

If the relays have statistical knowledge about first hop fading, it is possible to set $G$ to the average of the CSI-assisted variable relay gain [3] as follows:

$$
G^{2}=\mathbb{E}\left[\frac{\mathcal{E}_{2}}{\mathcal{E}_{1} \mathbf{h}_{S R_{l}}^{H} \mathbf{h}_{S R_{l}}+N_{0}}\right]=\int_{0}^{\infty} \frac{\mathcal{E}_{2}}{N_{0}\left(\gamma_{1}+1\right)} f_{\gamma_{1}}\left(\gamma_{1}\right) d \gamma_{1}
$$

Using (5) and [15, eqn.(3.383.10)] the gain parameter can then be solved as :

$$
\begin{array}{r}
G^{2}=\frac{\bar{\gamma}_{2} N_{t} !}{\left(N_{t s}-1\right) ! \bar{\gamma}_{1}^{N_{t s}}} \sum_{i=0}^{N_{t}-1} \frac{(-1)^{i}}{i !\left(N_{t}-1-i\right) !} \exp \left(\frac{(i+1)}{\bar{\gamma}_{1}}\right) \\
\cdot \sum_{j=0}^{i\left(N_{t s}-1\right)} \frac{\eta_{N_{t s}}(i, j)}{\bar{\gamma}_{1}^{j}} \boldsymbol{\Gamma}\left[j+N_{t s}\right] \boldsymbol{\Gamma}\left[1-j-N_{t s}, \frac{i+1}{\bar{\gamma}_{1}}\right]
\end{array}
$$

where $\Gamma[\cdot, \cdot]$ represents the incomplete Gamma function [15]. From (18), $C$ is obtained as $C=\frac{\mathcal{E}_{2}}{N_{0} G^{2}}$. Thus, this would make the performance of semi-blind relays comparable to that of CSI-assisted relays, while at the same time keeping implementation complexity low. The gain parameter may be periodically advised to the relays, as and when channel statistics changes.

\section{Statistics of the end-to-end SNR}

Here the closed form expression for $\mu_{n}$, the $n^{\text {th }}$ moment of the output SNR is derived. Important system measures such as average output SNR, variance or higher order central moments such as skewness and kurtosis parameters, can hence be obtained. These aid in comparison with other systems using a different fading model or where closed form solutions of BER or outage probability are not readily available. Higher order moments are also useful for further system analysis such as in SNR estimation[19]. In terms of the CDF, $\mu_{n}$ is given as:

$$
\mu_{n}=\mathbb{E}\left[\gamma_{e q}^{n}\right]=n \int_{0}^{\infty} \gamma_{e q}^{n-1}\left(1-F_{\gamma_{e q}}(\gamma)\right) d \gamma
$$

From (11), (19) is derived ${ }^{1}$ using [15, eqn.(6.631.3)] and after simplification results in:

$$
\begin{aligned}
\mu_{n} & =\frac{n N_{t} ! \exp \left(C / 2 \bar{\gamma}_{2}\right) \bar{\gamma}_{1}^{n}}{\left(N_{t s}-1\right) !\left(N_{r d}-1\right) !} \sum_{i=0}^{N_{t}-1} \frac{(-1)^{i}}{i !\left(N_{t}-1-i\right) !} \\
& \cdot \sum_{j=0}^{i\left(N_{t s}-1\right)} \frac{\eta_{N_{t s}}(i, j)\left(N_{t s}+j-1\right) !}{(i+1)^{N_{t s}+n+j}} \sum_{l=0}^{j+N_{t s}-1} \frac{\boldsymbol{\Gamma}[l+n]}{l !} \\
& \cdot \sum_{p=0}^{l}\left(\begin{array}{l}
l \\
p
\end{array}\right)\left(\frac{C}{\bar{\gamma}_{2}}\right)^{\frac{N_{r d}+p-1}{2}} \boldsymbol{\Gamma}\left[N_{r d}-p+l+n\right] \\
& \cdot \mathbf{W}_{-\left(2(l+n)+N_{r d}-p-1\right) / 2,\left(N_{r d}-p\right) / 2}\left(\frac{C}{\bar{\gamma}_{2}}\right)
\end{aligned}
$$

As a check, with $N_{t s}=N_{r d}=1$ and $N_{t}$ relays, (20) reduces to [7, eqn.(9)]. Next, we can derive the $n^{\text {th }}$ central moments about the mean as $\mu_{n}^{\prime}=\mathbb{E}\left[\left(\gamma_{e q}-\mathbb{E}\left[\gamma_{e q}\right]\right)^{n}\right]$ which is expanded as [14]: $\mu_{n}^{\prime}=\sum_{j=0}^{n} \frac{n !(-1)^{n-j} \mu_{j} \mu_{1}^{n-j}}{j !(n-j) !}$. Important third and fourth standardised moments such as the skewness (indicative of PDF asymmetry) and kurtosis (PDF peakiness) can be derived from this to gain further insight into the nature of the output SNR: the $n^{\text {th }}$ standardised moment is $\frac{\mu_{n}}{\mu_{2}^{\prime n / 2}}$.

\section{NUMERICAL AND SIMULATION RESULTS}

Next, outage probability and BER for BPSK are evaluated from these equations, and confirmed through simulation. For clarity, $C=\bar{\gamma}_{1}$ in all plots, so $G^{2}=\mathcal{E}_{2} / \mathcal{E}_{1}$. Fig. 2 plots the performance of relay selection in single and multiantenna terminals, with the dual-hop plot systems represented as $\left(N_{t}, N_{t s}, N_{r d}\right)$. The figure compares outage performance of several single antenna nodes and multi-antenna systems, with $\bar{\gamma}_{2}=5 \bar{\gamma}_{1}$ and a specific value of threshold $\gamma_{T}=5$. Clearly, a steep improvement can be obtained through the use of multiple antennas and relays. We see that the performance of $\left(N_{t}, 1,1\right)$ is relatively flat, and the effect of adding relays quickly diminishes; the gains being limited largely by the second hop channel, as noted in [7],[8] (single antenna case). With multiple antennas, large gains are observed even with a small number of relay terminals. Thus the use of multiple antennas is particulary beneficial when the number of relay nodes is limited. Another advantage is that when reverse channel bandwidth is limited it helps reduce CSI feedback load to the source as the relay nodes used for selection increase in number. Following information theoretic results in [20], the diversity order of this system will be $\min \left(N_{t} \times N_{t s}, N_{r d}\right)$, the minimum number of first hop and second hop diversity paths. With optimal relay selection, the effective number of forward diversity paths created are $N_{t} \times N_{t s}$, suggestive of

\footnotetext{
${ }^{1}$ The $n^{\text {th }}$ moment can be obtained from (16) as $\mathbb{E}\left[\gamma_{e q}^{n}\right]=$ $\left.(-1)^{n} \frac{d^{n}}{d s^{n}} \mathcal{M}_{\gamma_{e q}}(s)\right|_{s=0}$. However, it is easier to derive from (19).
} 


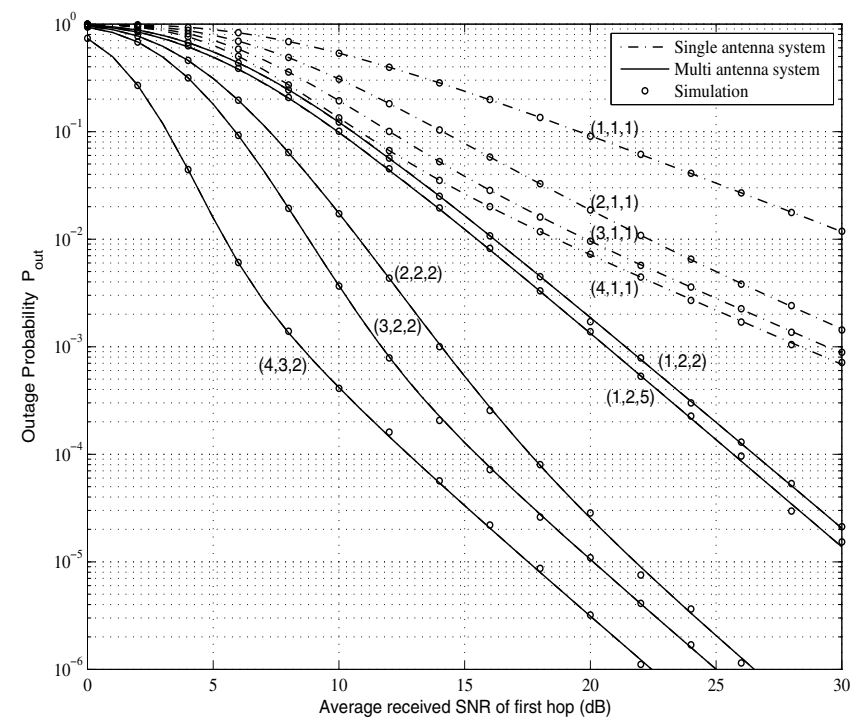

Fig. 2: Outage Probability for different relay configurations in the dual-hop multi-antenna scheme, with $\gamma_{T}=5, \bar{\gamma}_{2}=5 \bar{\gamma}_{1}$.

the diversity order of the first path, whereas, for the second hop, the diversity order is $N_{r d}$. Thus in the multi-antenna cases shown, the curves are found to have similar slopes at high SNR, and for the single antenna case of $\left(N_{t}, 1,1\right)$ the diversity order of the second hop channel determines performance at high SNR. It is also seen that if the quality of the first hop is better than the second hop (realised by multiple relays and transmit antennas) then large performance gains are possible, i.e, there is room for performance improvement by increasing $N_{r d}$ or $G$. However, if the opposite is true, or if the second hop link is comparable to the first, then performance saturates quickly, as seen in the $(1,2,2)$ setup: increasing $N_{r d}$ from two to five produced small improvement and further increase in $N_{r d}$ was found to offer almost no improvement. Similarly little improvement is seen by increasing $G$. However, adding an extra relay to the $(1,2,2)$ setup drastically improves performance, as observed from the $(2,2,2)$ outage curve. Fig. 3 plots the BER of $(5,1,1)$ and $(2,2,2)$ setups with different gains, where an increase in gain is beneficial to both systems. Note the total number of antennas in $(5,1,1)$ is one more than in $(2,2,2)$. Performance improvement is evident in the $(2,2,2)$ case over and above $(5,1,1)$, although we note that in reality, increasing $G$ beyond a certain point may not be profitable.

\section{CONCLUSION}

Closed form performance metric solutions have been derived for a wireless two-hop system with multi-antenna source and destination communicating via a single antenna AF bestrelay selected from a set of candidates. The source and destination employ beamforming and MRC respectively. The analytical solutions, along with simulations, have been used in one possible design space exploration: that of determining how best to allocate the system resource of additional antennas: in more relays, in more antennas at source, or more at destination. It was seen that careful selection of system parameters is important to realise the best improvement. It

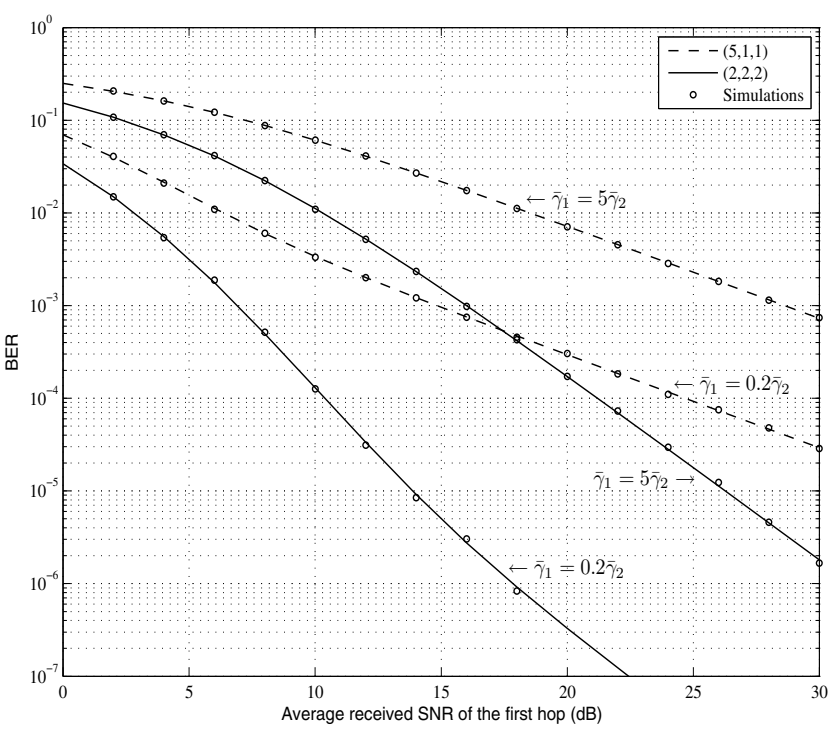

Fig. 3: BER performance for different antenna configurations and relay gains for BPSK modulation.

is worth noting two variants: (a) setting $N_{r d}=N_{t s}$ thus constitutes a symmetrical system of multi-antenna devices communicating with the assistance of a set of several simple single-antenna relay nodes, (b) that the equations presented also apply for the case of single antenna source/destination with multiple antenna relays (having MRC for receive and beamforming for transmit); a very different deployment scenario, but nevertheless also of potential practical relevance.

\section{REFERENCES}

[1] J. N. Laneman and G. W. Wornell, "Energy-efficient antenna sharing and relaying for wireless networks," in Proc. IEEE WCNC 2000, Oct. 2000, pp. 7-12 vol.1.

[2] R. Pabst, Pabst, Pabst, Pabst, and Pabst, "Relay-based deployment concepts for wireless and mobile broadband radio," IEEE Commun. Mag., vol. 42, no. 9, pp. 80-89, Sept. 2004.

[3] M. O. Hasna and M.-S. Alouini, "A performance study of dual-hop transmissions with fixed gain relays," IEEE Trans. Wireless Commun., vol. 3, no. 6, pp. 1963-1968, Nov. 2004.

[4] A. Bletsas, A. Khisti, D. P. Reed, and A. Lippman, "A simple cooperative diversity method based on network path selection," IEEE J. Sel. Areas Commun., vol. 24, no. 3, pp. 659-672, Mar. 2006.

[5] Y. Zhao, R. Adve, and T. J. Lim, "Symbol error rate of selection amplifyand-forward relay systems," IEEE Commun. Lett., vol. 10, no. 11, pp. 757-759, Nov. 2006.

[6] I. Krikidis, J. Thompson, S. McLaughlin, and N. Goertz, "Amplifyand-forward with partial relay selection," IEEE Commun. Lett., vol. 12, no. 4, pp. 235-237, Apr. 2008.

[7] H. Suraweera, D. Michalopoulos, and G. Karagiannidis, "Semi-blind amplify-and-forward with partial relay selection," vol. 45, no. 6, pp. 317-319, Mar. 2009.

[8] D. da Costa and S. Aissa, "End-to-end performance of dual-hop semiblind relaying systems with partial relay selection," IEEE Trans. Wireless Commun., vol. 8, no. 8, pp. 4306-4315, Aug. 2009.

[9] D. B. da Costa and S. Aissa, "Performance analysis of relay selection techniques with clustered fixed-gain relays," IEEE Signal Process. Lett., vol. 17, no. 2, pp. 201-204, Feb. 2010.

[10] H. Min, S. Lee, K. Kwak, and D. Hong, "Effect of multiple antennas at the source on outage probability for amplify-and-forward relaying systems," IEEE Trans. Wireless Commun., vol. 8, no. 2, pp. 633-637, Feb. 2009.

[11] D. da Costa and S. Aissa, "Cooperative dual-hop relaying systems with beamforming over Nakagami-m fading channels," IEEE Trans. Wireless Commun., vol. 8, no. 8, pp. 3950-3954, Aug. 2009.

[12] T. Lo, "Maximum ratio transmission," IEEE Trans. Commun., vol. 47, no. 10 , pp. 1458-1461, Oct. 1999. 
[13] D. Brennan, "Linear diversity combining techniques," in Proc. IEEE, vol. 91, no. 2, pp. 331-356, Feb. 2003.

[14] A. Papoulis and S. Pillai, Probability, Random Variables and Stochastic Processes, 4th ed. Boston, MA: McGraw-Hill, 2002.

[15] I. S. Gradshteyn and I. M. Ryzhik, Table of Integrals, Series, and Products, 6th ed. NY: Academic, 2000.

[16] A. Goldsmith, Wireless Communications. Cambridge, UK: Cambridge University Press, 2005.

[17] I. Wolfram Research, [Online]. Available: http://functions.wolfram.com/ 07.45.03.0009.01.
[18] M. Simon and M. Alouini, Digital Communication over Fading Channels: A Unified Approach to Performance Analysis, 1st ed. New York, NY: John Wiley and Sons, 2000, chapter 5.

[19] R. Matzner and F. Englberger, "An SNR estimation algorithm using fourth-order moments," in Proc. IEEE Intl. Symp. Inf. Theory, 1994, p. 119.

[20] M. Yuksel and E. Erkip, "Multiple-antenna cooperative wireless systems: A diversity;multiplexing tradeoff perspective," IEEE Trans. Inf. Theory, vol. 53, no. 10, pp. 3371-3393, Oct. 2007. 